\title{
Metodologia de rastreamento administrativo para segurança do paciente: um relato de experiência
}

\author{
Administrative tracking methology for patient safety:an experience report \\ Metodología administrativa de seguimento para la seguridad del paciente: un informe de \\ experiencia
}

Leisson Domingues Pinheiro ${ }^{1 *}$, Nazaré do Socorro Lopes de Souza1', Claudete Ferreira Quintino1, Paola Alejandra Valenzuela Reyes ${ }^{1}$, José Batista Luz Neto.

\section{RESUMO}

Objetivo: Relatar os benefícios da metodologia tracer ou rastreamento nos processos administrativos para identificar falhas em relação a identificação segura do paciente. Relato de Experiência: Trata-se de um estudo relato de experiência de um Centro integrado de Inclusão e Reabilitação do estado do Pará em relação a implantação do tracer administrativo e evidências dos seus benefícios nos processos de trabalho. $O$ tracer criou evidências acerca do uso da metodologia no setor administrativo, sobretudo quanto aos contextos de sua utilização e os resultados alcançados com diminuição de notificações de incidentes e desenvolvimento de todo o processo educativo e rastreamento no prontuário, apresentação e abordagens dos aspectos negativos e positivos para mediar conhecimentos que contribuem para efetivar o entendimento e as ações para identificação correta dos pacientes. Considerações Finais: O método assentou-se no caráter educativo por seu aspecto em demonstrar o problema, despertar o sensu crítico e reflexivo entre os colaboradores, opção como prática de melhoria na segurança do paciente.

Palavras chave: Educação, Segurança do paciente, Metodologia.

\begin{abstract}
Objective: To report the benefits of the tracer or tracking methodology in administrative processes to identify failures in relation to the safe identification of the patient. Experience report: This is an experience report study of an integrated Inclusion and Rehabilitation Center in the state of Pará in relation to the implementation of the administrative tracer and evidence of its benefits in the work processes. The tracer created evidence about the use of the methodology in the administrative sector, especially regarding the contexts of its use and the results achieved with the reduction of incident reports and the development of the entire educational process and tracking of medical records, presentation and approaches of negative and positive aspects. to mediate knowledge that contributes to effective understanding and actions for the correct identification of patients. Final Considerations: The method was based on the educational character due to its aspect in demonstrating the problem, awakening the critical and reflective sense among employees, an option as a practice to improve patient safety.
\end{abstract}

Keywords: Education, Patient safety, Methodology.

\section{RESUMEN}

Objetivo: informar los beneficios de la metodología de seguimiento o seguimiento en los procesos administrativos para identificar fallas en relación con la identificación segura del paciente. Informe de experiencia: Este es un estudio de informe de experiencia de un Centro integrado de inclusión y rehabilitación

${ }^{1}$ Centro Integrado de Inclusão e Reabilitação (CIIR/INDSH), Belém-PA. *E-mail: qualidade.ciir@indsh.org.br SUBMETIDO EM: 1/2020 
en el estado de Pará en relación con la implementación del trazador administrativo y la evidencia de sus beneficios en los procesos de trabajo. El trazador creó evidencia sobre el uso de la metodología en el sector administrativo, especialmente en relación con los contextos de su uso y los resultados logrados con la reducción de los informes de incidentes y el desarrollo de todo el proceso educativo y el seguimiento de los registros médicos, la presentación y los enfoques de aspectos negativos y positivos para mediar en el conocimiento que contribuye a la comprensión efectiva y las acciones para la identificación correcta de los pacientes. Consideraciones finales: El método se basó en el carácter educativo debido a su aspecto para demostrar el problema, despertando el sentido crítico y reflexivo entre los empleados, una opción como práctica para mejorar la seguridad del paciente.

Palabras clave: Educación, Seguridad del paciente, Metodología.

\section{INTRODUÇÃO}

O protocolo de identificação do paciente é um instrumento gerencial que deve instituir estratégias que garantem a adequada identificação dos pacientes. Nesse sentido, promove a segurança do paciente, uma vez que todos métodos de identificação afetam diretamente a segurança, colocando em risco iminente de um evento adverso (ALMEIDA HOC, SOUZA DAS, SANTOS FS ,2019). A identificação correta do paciente é o processo pelo qual assegura ao paciente que ele é destinado de determinado tipo de procedimento ou tratamento, prevenindo ocorrências de erros ou enganos que possam levar ao incidente. Os equívocos ocorridos na identificação são fatores preocupantes na assistência à saúde, já que um erro de identificação induz a uma série de eventos adversos (ALVES ECC, LIMA JLL, AMARO MOF, VIEIRA NC,2019).

As instituições de saúde têm investido cada vez mais em ações que visem à promoção da qualidade e segurança na assistência do paciente, com a inclusão de uma cultura de segurança e o primeiro passo para essa cultura começam com a identificação do paciente. O propósito da meta de identificação segura é identificar de modo confiável o indivíduo como sendo a pessoa para qual se destina o serviço ou tratamento e assegurar o procedimento a ser executado seja efetivo ao paciente (MAZIERO ECS, PEGORARO LGO, CABULON EAIC, VITURI DW, et al.,2019).

A Organização Mundial da Saúde (OMS) recomenda que as organizações de saúde façam a identificação correta do paciente e uso de pulseiras de identificação que comtemplam marcadores de identificação. Ressalta ainda, a importância de identificar as causas quando ocorrer algum incidente relacionado a identificação. Quando discutido as causas, as barreiras devem ser implantadas para evitar recorrências e minimizar os riscos para os pacientes. (WERNEK AL, RODRIGUES AMSR, FRUTUOSO IS, BECCARIA LM, et al., 2018).

A Organização Mundial da Saúde (OMS) recomenda que as organizações de saúde façam a identificação correta do paciente e uso de pulseiras de identificação que comtemplam marcadores de identificação. Ressalta ainda, a importância de identificar as causas quando ocorrer algum incidente relacionado a identificação. Quando discutido as causas, as barreiras devem ser implantadas para evitar recorrências e minimizar os riscos para os pacientes.

A identificação correta dos pacientes é a primeira meta Internacional de segurança do paciente, tendo de assegurar um tratamento seguro e reduz eventos adversos na administração de medicamentos, sangue ou hemoderivados, nas coletas de sangue ou outras amostras para exames clínicos, ou quaisquer outros tratamentos ou qualquer tipo de procedimento (MELGAÇO RMT e NEVES LAC, 2011). Os eventos adversos estão ligados diretamente ao erro humano que pode ter interferências que potencializam os riscos, por exemplo, condições de trabalho, estrutura física, complexidade da atividade laboral, deficiência de aperfeiçoamento dos recursos humanos mediante aos avanços tecnológicos, desmotivação e sobrecarga de trabalho (GONDIM MM; SAMPAIO RL; FIGUEIREDO SV, et al.,2014).

A identificação incorreta dos pacientes é capaz de comprometer o processo assistencial como um todo. A National Patiente Safety Agency (NPSA) constatou entre os anos de 2006 a 2007, 24.382 relatórios de situações incompatíveis com uma assistência segura aos pacientes, estima-se que desses, 2.900 estejam 
relacionados a erros de identificação como informações incorretas nas pulseiras e a não utilização das mesmas (ANDRADE GIS, 2018).

O método tracer é uma ferramenta sistemática de gestão para assegurar que as organizações de saúde possam rastrear as falhas dos processos e proporcionar cuidados mais seguros e de alta qualidade para os pacientes (BALDISSERA VDA, LABEGALINI MCG, MEIRELES VC, 2019).

$O$ tracer permite uma visão mais processual e permiti verificar as interfaces, pois o cuidado ocorre transversalmente, cada profissional da sua área atende o paciente em sequência e que nem sempre é harmoniosa, mostrando lacunas importantes, capazes de afetar a segurança do paciente e a qualidade dos cuidados. Esse método de rastreamento é amplamente utilizado nas avaliações da Joint Commission Internacional representa cerca de $60 \%$ a $70 \%$ das atividades realizadas durante seus processos avaliativos de acreditação (YAMAUCHI NI, 2016).

O presente estudo tem como objetivo relatar os benefícios da metodologia tracer ou rastreamento nos processos administrativos para identificar falhas em relação a identificação segura do paciente.

\section{RELATO DE EXPERIÊNCIA}

O estudo ocorreu em um centro de Integrado de Inclusão e Reabilitação (CIIR), localizado na cidade de Belém, capital do estado do Pará e atende 100\% dos usuários do Sistema Único de Saúde. Tem como negócio, reabilitação física, intelectual, auditiva, visual, oficina ortopédica, centro odontológico, ambulatório especializado e serviços auxiliares de diagnóstico e terapia.

Visto a relevância da temática procede com a descrição do protocolo de identificação segura com a participação multiprofissional e Núcleo da Qualidade e segurança do paciente para posterior validação e iniciação do processo de identificação para todos os usuários do centro, definindo com três marcadores (nome do paciente, nome da mãe e data de nascimento).

Após implantação das boas práticas de identificação, passamos a acompanhar as notificações dos incidentes relacionados a identificação. As ações de processo de implantação ocorreram nos meses de julho a agosto de 2019. Como norteador desse caminho entre a teoria e prática do processo de implantação da identificação segura a equipe multiprofissional e a lideranças da instituição teve um papel fundamental e estratégico. A equipe multiprofissional em parceria com as lideranças foi protagonista do protocolo, porém todo seu processo na prática envolveu vários colaboradores e parceiros, levando conhecimento a todos acerca da descrição e prática do protocolo de identificação segura. Com a finalidade de elaborar, planejar, implementar, acompanhar e alocar prazos voltados sempre a objetivos estratégicos que seria gerenciar com foco na segurança assistencial e elaboração de indicador taxa de adesão ao protocolo de identificação segura do usuário.

Foi definido a metodologia ativa de aprendizado baseado em problema (ABP) para servir como base de discussão após o rastreamento dos problemas identificados nos prontuários. As habilidades ABP a serem apreendidas são organizados em torno de um problema, havendo uma relação recíproca entre 0 conhecimento e o problema. O conhecimento é estimulado pelo o problema e aplicado de volta ao problema (ROCHA HM e LEMOS WM, 2014).

O Núcleo da qualidade e segurança do paciente (NQSP) realiza o monitoramento mensal das notificações de incidentes e o cumprimento semanal do tracer administrativo com os setores recepção, central interno de agendamento, serviço de prontuário do paciente e faturamento.

Durante o período de organização, o tracer teve seu início no dia 15 de outubro. Os envolvidos (auxiliares administrativos, supervisores e lideranças de áreas) adquiriram conhecimentos e habilidades que 0 capacitaram a desenvolver todo o processo educativo e rastreamento no prontuário, apresentação e abordagens dos aspectos negativos e positivos para mediar conhecimentos que contribuem para efetivar 0 entendimento e as ações para identificação correta dos pacientes.

As atividades são coordenadas pelo NQSP (Núcleo da qualidade e segurança do paciente) e a coordenadora administrativa do setor. São realizados nos prontuários 0 rastreamento e apresentados 
múltiplos aspectos de conformidades e não conformidades existentes. As apresentações ocorrem uma vez na semana e após à apresentação, são analisadas e discutidas ações de barreiras de prevenção do incidente. Todos as reuniões são registradas total de participantes.

Nesse sentido o próprio tracer criou evidências acerca do uso da metodologia no setor administrativo, sobretudo quanto aos contextos de sua utilização e os resultados alcançados. Com a realização sucessivas de reuniões e alinhamento dos objetivos, o entendimento da equipe começou a ser observados em relação a apresentação, resolutividade do problema e os indicadores para o monitoramento começaram a apresentarem resultados satisfatórios.

Gráfico 1 - Número de notificação de incidente - erro de identificação.

\section{Número de notificação de incidente \\ Erro de identificação}

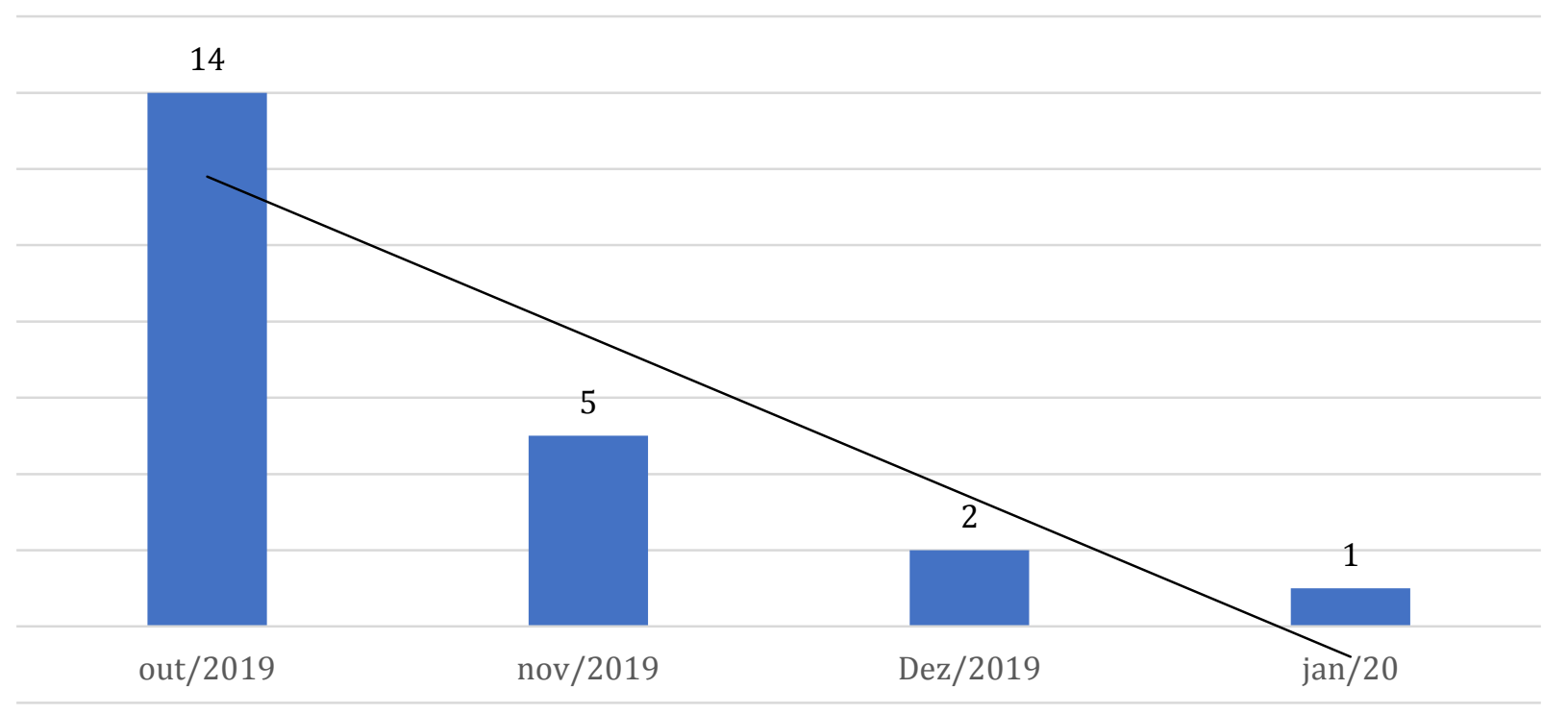

Fonte: Lapcchik MS, Ferraz RRN, Júnior SAPJ,2017.

A contribuição da metodologia no processo de formação e capacitação dos próprios colaboradores em identificar os pontos fracos nos quais o centro necessita melhorar. O tracer administrativo demonstrou um recurso pedagógico e uma ferramenta de gestão com mais vantagem que os métodos tradicionais. Os colaboradores participaram ativamente nas ações de melhorias.

\section{DISCUSSÃO}

Após aplicação do tracer administrativo nota-se uma redução gradativa dos registros relacionados a notificações de incidente erros de identificação entre os meses de novembro, dezembro e janeiro conforme gráfico 1. Entre as notificações de incidentes de erros de identificação, destacam-se redução de registros relacionados a erros no momento do cadastro dos usuários, como nome e sobrenome dos usuários errados, ausência do nome da mãe, data de nascimento errado, ausência de pulseira de identificação nos usuários, inserção errôneas de documentos em prontuários.

A qualidade e melhores resultados em saúde depende de uma estrutura adequada, organização do atendimento e definição de suas etapas em cadeia de processos, além do monitoramento de indicadores, sua análise crítica e ações para correção de não conformidade. A técnica de rastreamento de processo de atendimento de uma condição traçadora busca análise profunda da assistência, avalia múltiplos elementos, não conformidades e oportunidades de melhorias (LAPCCHIK MS, FERRAZ RRN, JÚNIOR SAPJ,2017). 
As não conformidades de identificação do paciente são apontadas na assistência como fator de grande relevância de segurança na assistência à saúde. A identificação incorreta induz a uma série de eventos adversos que envolvi administração de medicamento, procedimentos em geral, exames, entre outros. O uso de pulseiras de identificação reduz a taxa de erros, mas a falta de exatidão dos dados contidos pode causar confusão e aumentar risco (TRONCHIN DMR, BIANCHINI SM, LOURENÇÃO DCA, et al.,2013).

O Centro adota as medidas de identificação do paciente com a utilização de pulseira em todos os usuários atendidos e tem como práticas a dupla conferência em todos os procedimentos, verificando os marcadores. A identificação deve ser realizada mediante a apresentação de documento oficial com foto nítida e dados legíveis. Na ausência da pulseira todos os profissionais são responsáveis por providenciar imediatamente uma nova pulseira.

A identificação do paciente inicia pelo profissional da recepção no momento do cadastro do usuário, nome completo sem abreviaturas, data de nascimento e nome completo da mãe sem abreviatura. Ao colocar a pulseira, o profissional responsável deve solicitar que o mesmo confirme os dados antes e orientações para os usuários e acompanhantes sobre o protocolo.

A qualidade em saúde é o grau em que os serviços prestados ao paciente diminuem a probabilidade de resultados favoráveis e aumentam a probabilidade de resultados favoráveis. É uma definição verificada na literatura de múltiplas abordagens temáticas da segurança do paciente e políticas de qualidade em saúde (LAGUARDIA J, et al., 2013).

A segurança do paciente é uma dimensão da qualidade da assistência, sendo considerado a um mínimo aceitável do risco de dano desnecessário associado ao cuidado de saúde. É necessário o trabalho interdisciplinar integrado das equipes com apoio da alta direção, para que os esforços sejam concentrados para correções de processos inseguros (MALIK AM, PADILHA KG, BARBOSA AP, et al.,2016)

$O$ atendimento seguro ao paciente é resultado de diversas ações implantadas dentro das organizações de saúde. A cultura de segurança evidenciada, estabelecimento de metas, medidas e protocolos pontuais, como a identificação do paciente, são estratégias para redução dos incidentes de segurança conforme o Programa Nacional de Segurança do Paciente (PNSP) (NICOLA LA, RIGO DFH, ALVES SR, et al., 2016).

As organizações de saúde que buscam melhoria contínuas em seus processos de trabalhos, devem assegurar e garantir adesão aos seus protocolos de segurança do paciente, minimizando riscos à saúde do paciente. O resultado é a relação harmoniosa das dimensões gerenciais, econômica, tecnológica, assistencial, de ensino e pesquisa (TRONCHIN DMR e QUADRADO ERS, 2012).

Observa-se que mesmo as organizações de saúde façam a construção dos protocolos de segurança do paciente, ainda há grandes dificuldades de efetivar e monitorar protocolo de identificação do paciente dos profissionais de saúde, gestores, entidade de classe e usuário. Motivo no qual, o assunto vem conquistando espaços e dimensões para discussões e alternativas para reciclagem e periodicidade de treinamentos para que sua prática se torna uma cultura organizacional de responsabilidade de todos envolvidos, bem como a mensuração, monitoramento dos resultados e divulgação dos resultados para reduzir os riscos (NADAI TR, SOUZA DAS, MENEZES MS, et al., 2012).

O tracer vem demostrando uma metodologia que fortalece essas barreias para evitar esses eventos adversos, pois desperta nos colaboradores o incentivo em identificar as próprias falhas e cria ações para melhorias nos processos.

O Tracer interfere positivamente nos processos administrativos que estejam ligados diretamente na identificação segura dos usuários. O método avaliativo, permite listar as não conformidades e as conformidades com os padrões estabelecidos na instituição relacionados a segurança do paciente.

O método assentou-se no caráter educativo por seu aspecto em demonstrar o problema, despertar o sensu crítico e reflexivo entre os colaboradores, opção como prática cotidiana no trabalho. Sugere-se estudo posteriores que ampliem a busca para publicações relacionados a metodologias de avaliação em serviços de saúde afim de conhecer mais instrumentos utilizados na qualidade e segurança do paciente. 


\section{REFERÊNCIAS}

1. ALMEIDA HOC, et al. Protocolo de identificação e a interface com a segurança do paciente. Ciências Biológicas e de Saúde.2019;5(3):27-40.

2. ALVES ECC, et al. A identificação do paciente no alcance de práticas seguras: concepções e práticas. Revista enfermagem atual.2019;87.

3. MAZIERO ECS, et al. Identificação segura do paciente: adequação do uso da pulseira por impressão térmica em um hospital público universitário do norte do Paraná. Saúde Pública.2019;2(1):11-20.

4. WERNEK AL, et al. Adesão ao protocolo de identificação do paciente e medicação segura. Revista de Enfermagem UFPE.2018;12(10):2667-2674.

5. MELGAÇO RMT, NEVES LAC. A identificação do paciente como indicador de qualidade. Universidade Federal do Estado do Rio de Janeiro.2011.88-100.

6. GONDIM MM, et al. Estratégia para promover segurança do paciente: da identificação dos riscos às práticas baseadas em evidências. Revista de enfermagem Ana Nery.2014:18(1);122-129.

7. ANDRADE AGI. Segurança do paciente no cuidado a criança hospitalizada: práticas de enfermagem relacionadas à identificação do paciente. Trabalho de conclusão de curso (bacharelado em enfermagem). Centro de formação de professores. Universidade Federal de Campina Grande, Cajazeiras,2018;60 p.

8. BALDISSERA VDA, et al. Método tracer e a qualidade do cuidado na enfermagem: revisão bibliográfica. Revista Integrativa.2019;40:1-12.

9. ANDRADE AGI. Segurança do paciente no cuidado a criança hospitalizada: práticas de enfermagem relacionadas à identificação do paciente. Trabalho de conclusão de curso (bacharelado em enfermagem). Centro de formação de professores. Universidade Federal de Campina Grande, Cajazeiras,2018;60 p.

10. YAMAUCH NI, et al. A metodologia do consórcio brasileiro de acreditação. Revista Baiana de Saúde Pública.2016;1:286-294.

11. ROCHA HM, LEMOS WM. Metodologias ativas: do que estamos falando? Base conceitual e relato de pesquisa em andamento. Simpósio pedagógico e pesquisa em educação.2014:2041-201412.

12. LAPCCHIK MS, et al. Metodologia da condição traçadora para avaliação do processo assistencial de pacientes com sepse grave e choque séptico. Revista de gestão em sistema de saúde.2017:6(2);114-123.

13. TRONCHIN DMR, et al. Identificação do paciente nas organizações de saúde: uma reflexão emergente. Revista Gaúcha.2013;34(2):196-200.

14. LAGUARDIA J, et al. A segurança do paciente como dimensão da qualidade do cuidado de saúde um olhar sobre a literatura.2013:18(7);2012-2036.

15. MALIK AM, et al. Segurança do paciente na cultura organizacional: percepção das lideranças de instituições hospitalares de diferentes natureza administrativas. Revista da Escola Enfermagem da USP.2016:50(3);490-497.

16. NICOLA LA, RIGO DFH, ALVES SR, et al. Qualidade da assistência e segurança do paciente: avaliação por indicadores. Cogitare Enfermagem.2016:21;1-8.

17. TRONCHIN DMR, QUADRADO ERS. Avaliação do protocolo de identificação do neonato de um hospital privado. Revista Latina Americana de Enfermagem.2012:20(4);1-8.

18. NADAI TR, et al. A implantação do protocolo de identificação segura como ferramenta de segurança do paciente. Revista QualidadeHC.2012: 14-20. 